\title{
Accounting for residual activity in the estimate of myocardial blood flow with PET
}

\author{
Jonathon A. Nye, PhD, ${ }^{a}$ and C. David Cooke ${ }^{a}$ \\ a Department of Radiology and Imaging Sciences, Emory School of Medicine, Emory University, \\ Atlanta, GA
}

Received Nov 7, 2021; accepted Nov 8, 2021

doi: $10.1007 / \mathrm{s} 12350-021-02873-7$

\section{See related article, pp. 2262-2270}

Estimation of myocardial blood flow (MBF) has many positives for patient care but the data collection and computation of flow are difficult to do well. The technical challenges of dynamic PET imaging for flow are many and may depend on the radiotracer employed, ${ }^{1}$ administration method, ${ }^{2}$ stress protocol, ${ }^{3}$ settings of the imaging system, ${ }^{4}$ motion management, ${ }^{5}$ and choice of kinetic model. ${ }^{6}$ The ideal radiotracer should have a high first-pass extraction that is not dependent on flow. A non-linear dependence leading to a roll-off in extraction at high flows, particularly for [Rb-82] chloride, puts further pressure on the user to ensure technical factors and corrections are accurate to avoid unintentionally amplifying measurement bias. Secondly, the radioactive label must have a sufficiently long half-life such that the radiotracer equilibrates or is trapped in the myocardium with sufficient counts to achieve good image quality and contrast compared with blood pool. Historically, clinical determination of MBF with PET has been limited to two radiotracers with markedly different extraction fractions and half-lives as well as their own logistical challenges in obtaining the agents.

[Rb-82] chloride has the shortest half-life with the convenience of being on-demand from a generator. These two advantages permit a rest/stress exam to be conducted in rapid succession. At the end of the rest study, nearly all activity from the administration has

Reprint requests: Jonathon A. Nye, PhD, Department of Radiology and Imaging Sciences, Emory School of Medicine, Emory University, 1841 Clifton Rd. NE, Atlanta, GA 30329; jnye@emory.edu

J Nucl Cardiol 2022;29:2271-3.

$1071-3581 / \$ 34.00$

Copyright ( 2021 The Author(s) under exclusive licence to American Society of Nuclear Cardiology decayed leaving a "clean" patient state for the stress administration. However, other challenges remain such as the need to administer relatively high amounts of radioactivity such that sufficient counts are collected following blood pool clearance. ${ }^{7}$ [N-13] Ammonia has a higher extraction than [Rb-82] chloride, particularly at high flows, and a longer half-life resulting in improved count statistics. The major barrier with [N-13] ammonia is the need for a cyclotron to be within a reasonable distance to produce individual or bulk doses sufficient for a rest and stress exam. [N-13] ammonia may also be conducted in short succession although the logistics of timing the delivery of [N-13] ammonia tends to introduce delays, particularly if individual doses are arriving between rest and stress studies. This may be overcome in part with a dedicated $\mathrm{N}-13$ production system $^{8}$ but with the caveat that when the delay between rest and stress administrations shortens, radioactivity may be present in the heart from the previous administration. Finally, the initial reporting of [F-18] flurpiridaz has been encouraging given its high extraction at high flows and long half-life that effectively de-couples the user from the costs and management of the radioisotope production pipeline and permits the shipment of single doses. Although this could be a benefit to wider clinical use of $\mathrm{MBF}$, the consequence is that meaningful amounts of radioactivity from the rest administration will be remaining in the myocardium during the stress administration. Thankfully, quantifying repeated administration of long-lived perfusion radiotracers has a breadth of literature support and techniques developed for 1-day [Tc-99m] sestamibi have been adopted in the processing of same-day [F-18] flurpiridaz. ${ }^{9,10}$ What remains to be answered is how a same-day protocol, where residual radioactivity remains in the myocardium, impacts the calculation of myocardial blood flow?

To address this question, we can start with reviewing the kinetic model assumptions. Of the numerous approaches available to estimate $\mathrm{MBF}$, all fall 
into two primary categories: (1) a first-pass model that includes a reversible compartment and (2) a retention model (e.g., microsphere analog). The latter is the most simplistic in implementation and has been proposed for [F-18] flurpiridaz ${ }^{11}$ while the former is typically employed for [Rb-82] chloride and [N-13] ammonia $^{12,13}$ but not in all cases. ${ }^{14,15}$ What's important to note is that all model approaches share one common assumption, there is no radioactivity in the system at the time of radiotracer administration. In the cases described above for successive administration of [N-13] ammonia or [F18] flurpiridaz, blood pool radioactivity concentrations are typically negligible at the time of second administration; however, substantial radioactivity may remain in the myocardium. ${ }^{16,17}$

To understand the magnitude of residual activity on MBF, we propose a simple simulation consisting of two successive radiotracer administrations of the same amount. Figure. 1A displays a decay-corrected flow curve from the first administration with no prior radioactivity in the system and the computed flow values using a first pass and a retention model. In Figure 1B, $20 \%$ of the radioactivity that perfused the heart in the first administration remains in the myocardium at the time of the second administration and the flow values are recomputed. When comparing the scenarios in Figure 1A and B we find that there is roughly a $19-21 \%$ increase in MBF for the second administration. Therefore, when residual activity is on-board in the myocardium, the calculation of MBF will be biased high and this result is a consequence of the modeling assumption that the system's initial state has no activity, which is violated in Figure 1B.

A

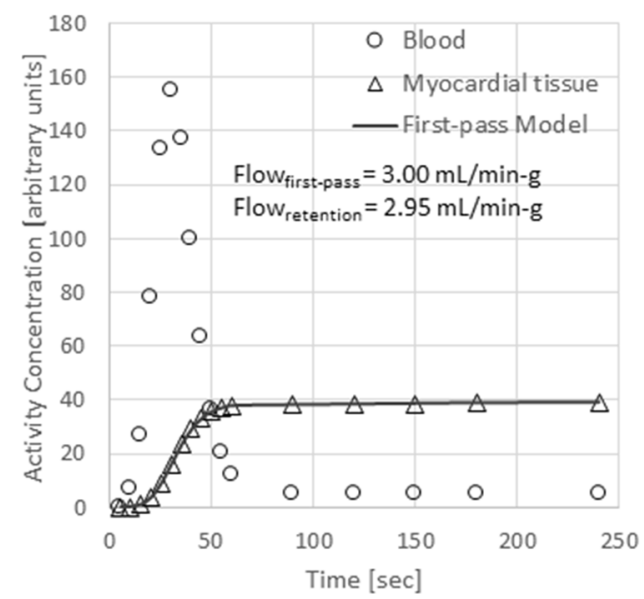

The experimental support to the hypothesis that residual radioactivity in the myocardium biases MBF is presented by Poitrasson-Riviere et al. ${ }^{18}$ for the two flow agents, [N-13] ammonia, and [F-18] flurpiridaz. Their team expertly demonstrate that residual radioactivity in the myocardium leads to an upward bias in the calculation of $\mathrm{MBF}$ and this bias increases with higher residual radioactivity on-board (see Figure $2 \mathrm{a}$ and $\mathrm{b}$ in Ref. 18). Their proposed solution is to measure the residual activity in the heart by collecting data a few minutes prior to the second radiotracer administration. The residual radioactivity is then subtracted from the myocardial tissue regions of interest prior to the postprocessing of the second scan. This practical approach adds negligible complexity to the dynamic PET protocol and preserves the assumptions of the kinetic model. Furthermore, Poitrasson-Riviere and colleagues provide Figure 1B to assist the reader in design of their protocol that includes (1) how the time delay between the rest and stress administrations relates to overestimation of MBF and (2) how increasing the amount of activity administered at stress compared to rest can reduce overestimation in MBF.

Given the large number of challenges to estimating MBF with PET, Poitrasson-Riviere et al. ${ }^{18}$ have provided a straightforward approach to manage the problem of residual radioactivity. With the anticipation of [F-18] flurpiridaz entering the clinic, this agent along with centers implementing [N-13] ammonia imaging with short delays should carefully scrutinize their image processing tools and software purchases for features that account for residual activity. As demonstrated by the authors, it will be important how the administration

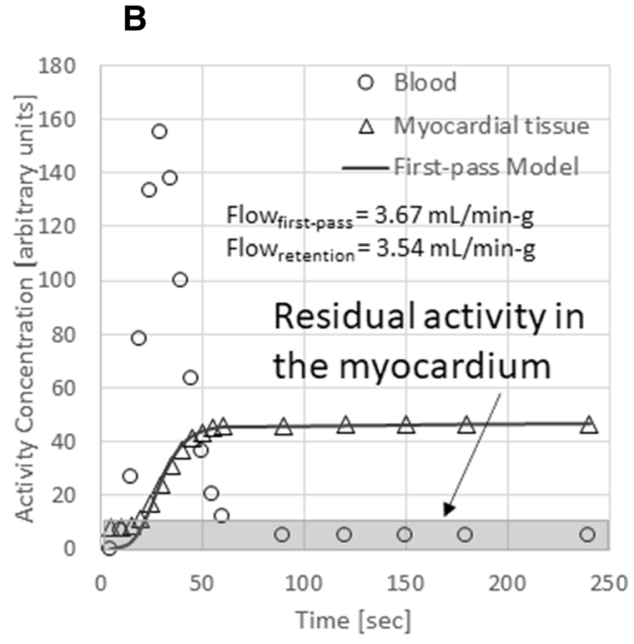

Figure 1. A simulation of flow with (A) no residual activity on-board and (B) $20 \%$ residual activity in the myocardium as indicated by the shaded area. The calculated flow values for each case are presented for first pass and retention models. 
protocol is setup and how residual activity is addressed in post-processing to ensure the best accuracy and precision in calculation of MBF.

\section{Disclosures}

C. David Cooke receives royalties from the sale of the Emory Cardiac Toolbox and has equity positions with Syntermed, Inc. The terms of these arrangements have been reviewed and approved by Emory University in accordance with its conflict of interest policies.

\section{References}

1. Murthy VL, Bateman TM, Beanlands RS, Berman DS, BorgesNeto S, Chareonthaitawee P. Clinical quantification of myocardial blood flow using PET: Joint position paper of the SNMMI cardiovascular council and the ASNC. J Nucl Med 2018;59:273-93.

2. Klein R, Ocneanu A, Renaud JM, Ziadi MC, Beanlands RSB, de Kemp RA. Consistent tracer administration profile improves testretest repeatability of myocardial blood flow quantification with (82)Rb dynamic PET imaging. J Nucl Cardiol 2018;25:929-41.

3. Kitkungvan D, Lai D, Zhu H, Roby AE, Johnson NP, Steptoe DD, et al. Optimal adenosine stress for maximum stress perfusion, coronary flow reserve, and pixel distribution of coronary flow capacity by Kolmogorov-Smirnov analysis. Circ Cardiovasc Imaging 2017. https://doi.org/10.1161/CIRCIMAGING.116. 005650 .

4. Armstrong IS, Tonge CM, Arumugam P. Impact of point spread function modeling and time-of-flight on myocardial blood flow and myocardial flow reserve measurements for rubidium- 82 cardiac PET. J Nucl Cardiol 2014;21:467-74.

5. Memmott MJ, Tonge CM, Saint KJ, Arumugam P. Impact of pharmacological stress agent on patient motion during rubidium82 myocardial perfusion PET/CT. J Nucl Cardiol 2018;25:128695.

6. Choi Y, Huang SC, Hawkins RA, Kim JY, Kim BT, Hoh CK. Quantification of myocardial blood flow using $13 \mathrm{~N}$-ammonia and PET: Comparison of tracer models. J Nucl Med 1999;40:1045-55.

7. Bateman TM, Heller G, Mcghie A, Friedman J, Case J, Bryngelson J, et al. Diagnostic accuracy of rest/stress ECG-gated Rb-82 myocardial perfusion PET: Comparison with ECG-gated Tc-99m sestamibi SPECT. J Nucl Cardiol 2006;13:24-33.

8. Pieper J, Patel VN, Escolero S, Nelson JR, Poitrasson-Rivière A, Shreves CK, et al. Initial clinical experience of N13-ammonia myocardial perfusion PET/CT using a compact superconducting production system. J Nucl Cardiol 2021;28:295-9.

9. Heo J, Powers J, Iskandrian AE. Exercise-rest same-day SPECT sestamibi imaging to detect coronary artery disease. J Nucl Med 1997;38:200-3.

10. Maddahi J, Lazewatsky J, Udelson JE, Berman DS, Beanlands RSB, Heller GV, et al. Phase-III clinical trial of fluorine-18 flurpiridaz positron emission tomography for evaluation of coronary artery disease. J Am Coll Cardiol 2020;76:391-401.

11. Packard RR, Huang S-C, Dahlbom M, Czernin J, Maddahi J. Absolute quantitation of myocardial blood flow in human subjects with or without myocardial ischemia using dynamic flurpiridaz $\mathrm{F}$ 18 PET. J Nucl Med 2014;55:1438-44.

12 Lortie M, Beanlands RSB, Yoshinaga K, Klein R, Da Silva JN, de Kemp RA. Quantification of myocardial blood flow with $82 \mathrm{Rb}$ dynamic PET imaging. Eur $\mathrm{J}$ Nucl Med Mol Imaging 2007;34:1765-74.

13. DeGrado TR, Hanson M, Turkington T, Delong D, Brezinski D, Vallee J, et al. Estimation of myocardial blood flow for longitudinal studies with $13 \mathrm{~N}$-labeled ammonia and positron emission tomography. J Nucl Cardiol 1996;3:494-507.

14. Yoshida K, Mullani N, Gould KL. Coronary flow and flow reserve by PET simplified for clinical applications using rubidium-82 or nitrogen-13-ammonia. J Nucl Med 1996;37:1701-12.

15 Nekolla SG, Reder S, Saraste A, Higuchi T, Dzewas G, Preissel A, et al. Evaluation of the novel myocardial perfusion positronemission tomography tracer 18F-BMS-747158-02: Comparison to $13 \mathrm{~N}$-ammonia and validation with microspheres in a pig model. Circulation 2009;119:2333-42.

16. Maddahi J, Czernin J, Lazewatsky J, Huang S-C, Dahlbom M, Schelbert H, et al. Phase I, first-in-human study of BMS747158, a novel 18F-labeled tracer for myocardial perfusion PET: Dosimetry, biodistribution, safety, and imaging characteristics after a single injection at rest. J Nucl Med 2011;52:1490-8.

17. Yi C, Yu D, Shi X, He Q, Zhang X, Zhang X. Biodistribution and estimation of radiation-absorbed doses in humans for $13 \mathrm{~N}$-ammonia PET. Ann Nucl Med 2015;29:810-5.

18. Poitrasson-Rivière A, Moody JB, Renaud JM, Hagio T, AridaMoody L, Buckley $\mathrm{C}$, et al. Impact of residual subtraction on myocardial blood flow and reserve estimates from rapid dynamic PET protocols. J Nucl Cardiol 2021. https://doi.org/10.1007/s123 50-021-02837-x.

Publisher's Note Springer Nature remains neutral with regard to jurisdictional claims in published maps and institutional affiliations. 